\title{
ANÄLISE COMPARADA DE CRESCIMENTO DO ENXERTO E DO PORTA-ENXERTO DE Hevea brasiliensis Muell. Arg.
} EM VIVEIRO

\author{
P.R.C. Castro* \\ L.M. Barbosa** \\ V.D.F. Nastri** \\ A.A. Lucchesi*
}

RESUMO: O objetivo do presente trabalho foi o de verificar a compatibilidade entre o enxerto ('RRIM $600^{\prime}$ ) e o porta-enxerto ('Tjir 16') de seringueira, atra vés da anälise comparada de crescimento. As plantas fōo ram cultivadas em recipientes plásticos, nas condiçōes de viveiro, em Piracicaba (SP). As amostras foram coletadas em 4 períodos (de duas épocas) com intervalos de 30 dias. Os valores da TAL da seringueira foram de 0,018 a $0,031 \mathrm{~g} \cdot \mathrm{dm}^{-2} \cdot \mathrm{dia}^{-1}$, da TCR de 0,0145 a $0,0165 \mathrm{~g} \cdot \mathrm{g}^{-1} \cdot \mathrm{dia}^{-1}$ e da RAF de 0,4363 a $0,8510 \mathrm{dm}^{2} \cdot \mathrm{g}^{-1}$. A VPS e a VAF revelaram um maior vigor do porta-enxerto com relação ao enxerto e uma certa incompatibilidade no período de desenvolvimento de 'Tjir 16 ' em relação ao 'RRIM 600'. A RAF e a RPF mostraran, respectivamente, uma maior proporção relativa da ārea e do peso foliar no peso total da planta no início do desenvolvimento do enxerto e mais tardiamente no porta-enxerto. Verificouse uma relação direta entre os valores da TCR e da TAL do enxerto e do porta-enxerto, sendo que os cultivares não apresentaram diferenças sensiveis nos incrementos de

* Departamento de Botânica da E.S.A. "Luiz de Queiroz" da Universidade de São Paulo - 13.400 - Piracicaba, SP.

** Seção de Ecologia do Instituto de Botânica - 01.000Sao Paulo, SP, Bolsista do CNPq. 
matéria seca por unidade de tempo.

Termos para indexação: seringueira, anālise de crescimento, enxerto, porta-enxerto, compatibilidade.

COMPARED GROWTH ANALYSIS OF STOCK AND SCION OF Hevea brasiziensis Muell. Arg. IN NURSERY

ABSTRACT: An experiment was carried out to establish a comparative growth analysis of stock and scion of rubber plant under nursery conditions. Samples were collected in four periods at 30 days intervals. The results indicated values of 0.018 to $0.031 \mathrm{~g} \cdot \mathrm{dm}^{-2} \cdot \mathrm{day}^{-1}$ for NAR, 0.0145 to $0.0165 \mathrm{~g} \cdot \mathrm{g}^{-1} \cdot \mathrm{day}^{-1}$ for RGR and 0.4363 to $0.8510 \mathrm{dm}^{2} \cdot \mathrm{g}^{-1}$ for LAR of RRIM 600 and $\mathrm{Tj}$ ir 16 rubber cultivars. Dry weight variation and leaf area variation showed a higher vigour of the rootstock compared to the clone, and a difference in the growth period of 'Tjir $16^{\prime}$ compared with 'RRIM 600'. LAR and LWR showed, respectively, a higher relative proportion of leaf area and leaf weight in the dry weight of the plant in the initial phase of growth of the stock and latter in the clone. A direct relationship was noted between the values of RGR and NAR of the stock and clone. 'Tjir 16' and 'RRIM $600^{\prime}$ did not present sensible differences in dry matter increase with time.

Index terms: rubber, growth analysis, stock, scion.

\section{INTRODUÇAO}

Considerando-se o aumento da ärea cultivada e a precocidade que se tem conseguido para a seringueira, que normalmente inicia sua produção entre o 79 e 89 ano 
de idade, torna-se um imperativo a utilização de novas tecnologias de cultivo, capazes de possibilitar um aumento da produtividade dos seringais. Alia-se a isto - fato de ser o Brasil um grande importador de borracha, mesmo possuindo condições edafoclimáticas adequadas ao desenvolvimento e expansão da heveicultura.

A uniformidade do desenvolvimento das plantas de seringueira, representa garantia de alta produtividade, pois permite a obtenção do nümero adequado de plantas por ärea, com crescimento ideal por ocasião de início da produção de 1átex. Isto permitiria maximizar o aproveitamento da área onde a energia solar teria sua me lhor utilização devido a cobertura ōtima obtida atraves do dossel foliar gerado pela uniformidade no desenvolvimento das plantas.

GUTIERREZ \& JIMENEZ (1967) preconizaram a possibilidade de que os resultados da anālise de crescimento efetuada com plantas jovens de cacaueiro possam indicar com bastante precisão o desempenho produtivo futuro dos diferentes cultivares. Isto permitiria efetuar um descarte das plantas menos promissoras de seringueira, principalmente se os parâmetros da análise de crescimento forem complementados com a determinação precoce da produção de látex.

o estabelecimento dos parāmetros da anālise de crescimento possibilitaria a identificação da compatibilidade do enxerto com o porta-enxerto se as determinações forem comparadas e simultâneas.

$\mathrm{Na}$ formação de viveiros, nas condições brasileiras, geralmente são utilizadas sementes advindas de seringais nativos ou de misturas de clones que concorrem para que haja grande heterogeneidade nos porta-enxertos, contribuindo assim, para a redução do nümero de plantas a serem utilizadas na enxertia. Além disso, podem ocorrer casos de incompatibilidade entre o enxerto e o porta-enxerto que irão aumentar a heterogeneidade do plantio definitivo e prolongar o período de imaturidade do seringal (VALOIS et alii, 1978). 
Vários ensaios têm demonstrado que o vigor e a produtividade dos clones variam em função das caracteristicas dos porta-enxertos (MORAES \& VALOIS, 1979). Em países onde a cultura da seringueira è tecnicamente mais adiantada, jâ são conhecidas as melhores combinações entre clone e porta-enxerto oriundos de sementes clonais ilegítimas. Poucos são os trabalhos sobre a associação de clones com porta-enxertos em nossas condições, sendo que SANTOS (1982) considerou que os clones independem dos porta-enxertos utilizados, quanto ao desempenho.

o objetivo do presente trabalho foi o de verificar a compatibilidade entre o enxerto ('RRIM 600') e o porta-enxerto ('Tjir 16') de seringueira, através da análise comparada de crescimento.

\section{MATERIAL E METODOS}

Este experimento foi realizado em condições de viveiro, no Horto Experimental do Departamento de Botânica da E.S.A. "Luiz de Queiroz" em Piracicaba (SP). As sementes do porta-enxerto (Hevea brasiziensis cv. Tjir 16) foram colocadas no germinador em 15/05/83, ten do sido repicadas para recipiente plástico com capacidade para 81 itros $(40 \times 25 \mathrm{~cm})$, em $15 / 06 / 83$. 0 substra to utilizado constou de $2 / 3$ de terra argilosa e $1 / 3$ de terra arenosa, sendo que para cada $\mathrm{m}^{3}$ de substrato adicionou-se $1.800 \mathrm{~g}$ de superfosfato simples, $650 \mathrm{~g}$ de calcáreo e $250 \mathrm{~g}$ de cloreto de potássio. Procederam-se pos teriormente a 2 adubações com uréia em cobertua ( $3 \mathrm{~g} / \mathrm{re}-$ cipiente) e a 4 pulverizações mensais de nitrofoska. Em 15/01/84 foram coletadas amostras de terra dos recipien tes para anālise química. A anālise mostrou $\mathrm{pH}=5,0$, $\mathrm{C}=0,85 \%, \mathrm{P}=4 \mathrm{ppm}, \mathrm{K}=40 \mathrm{ppm}, \mathrm{Ca}=0,48 \mathrm{meq} / 100 \mathrm{~g}$, $\mathrm{Mg}=0,08 \mathrm{meq} / 100 \mathrm{~g}, \mathrm{Al}=1,42 \mathrm{meq} / 100 \mathrm{~g}, \mathrm{H}=5,47 \mathrm{meq} / 100 \mathrm{~g}$, $\mathrm{Cu}=2,2 \mathrm{ppm}, \mathrm{Fe}=12,9 \mathrm{ppm}, \mathrm{Mn}=0,80 \mathrm{ppm}$ e $\mathrm{Zn}=2,38 \mathrm{ppm}$.

As plantas enxertadas foram obtidas pela semeadura do porta-enxerto 'Tjir 16 ' no germinador em 28/02/81 
tendo sido repicadas para recipiente plástico em $28 / 03 / 81$. O substrato utilizado foi semelhante ao anterior, tendo recebido os mesmos insumos. A enxertia de Hevea brasiziensis cv. RRIM 600, nesses porta-enxertos, foi realizada em 25/10/82. Em 15/01/84 foram coletadas amostras de terra dos recipientes para análise química. A análise revelou $\mathrm{pH}=5,7, \mathrm{C}=0,78 \%, \quad \mathrm{P}=44 \mathrm{ppm}$, $\mathrm{K}=107 \mathrm{ppm}, \quad \mathrm{Ca}=2,64 \mathrm{meq} / 100 \mathrm{~g}, \quad \mathrm{Mg}=0,72 \mathrm{meq} / 100 \mathrm{~g}$, $\mathrm{A} 1=0,04 \mathrm{meq} / 100 \mathrm{~g}, \mathrm{H}=4,08 \mathrm{meq} / 100 \mathrm{~g}, \quad \mathrm{Cu}=2,06 \mathrm{ppm}$, $\mathrm{Fe}=20,36 \mathrm{ppm}, \mathrm{Mn}=1,58 \mathrm{ppm}$ e $\mathrm{Zn}=2,04 \mathrm{ppm}$.

A produtividade primária da seringueira foi determinada através de análise de crescimento de plântulas uniformes a partir do estabelecimento periódico de área foliar e do peso da matéria seca, das plântulas em período formativo. A partir desses valores foram então determinados os parâmetros da anālise de cres cimento correspondentes à variação de peso da matériáa seca $(V P S)=(P 2-P 1)$ em $g$, variação de área foliar $(\mathrm{VAF})=(\mathrm{A} 2-\mathrm{Al}) \mathrm{em} \mathrm{dm}^{2}$, ärea foliar específica $(\mathrm{AFE})=\mathrm{A} / \mathrm{P}$ foliar em $\mathrm{dm}^{2} \cdot \mathrm{g}^{-1}$, razão de área foliar $(\mathrm{RAF})=\mathrm{A} / \mathrm{P}$ em $\mathrm{dm}^{2} \cdot \mathrm{g}^{-1}$, razão de peso foliar $(\mathrm{RPF})=$ Pfo liar $/ \mathrm{P}$ em $\mathrm{g} \cdot \mathrm{g}^{-1}$, taxa de crescimento relativo $(\mathrm{TCR})=(\mathrm{LP} 2-\mathrm{LP} 1) /(\mathrm{T} 2-\mathrm{T} 1)$ em $\mathrm{g} \cdot \mathrm{dm}^{-2} \cdot \mathrm{dia}^{-1}$ e taxa assimilatória 1íquida (TAL) $=(\mathrm{P} 2-\mathrm{P} 1)(\mathrm{LA} 2-\mathrm{LA} 1) /(\mathrm{A} 2-\mathrm{A} 1)(\mathrm{T} 2-\mathrm{T} 1)$ em $\mathrm{g} \cdot \mathrm{dm}^{-2} \cdot \mathrm{dia}^{-1}$ (ALVIM, 1962).

Para as determinações destes parâmetros, foram utilizadas 36 plantas uniformes e em formação nos diferentes períodos de amostragem $(18+18$ plantas $)$. Estas eram avaliadas com intervalos médios de 30 dias e utilizando-se de técnicas apropriadas a partir da ärea foliar e peso da matéria seca nos diferentes períodos de amostragem e que foram 4 a saber: de 08/12/1983 a $12 / 01 / 1984$ (primeiro); de 12/01/1984 a 16/02/1984 (segundo); de $16 / 02 / 1984$ a $22 / 03 / 1984$ (terceiro) e 22/03/1984 a 26/04/1984 (quarto).

o número total de plantas utilizadas foi de 72 por período, sendo 36 de plantas originárias de sementes e 36 de plantas enxertadas ('RRIM 600'). Os resultados foram plotados em histogramas para sua anālise comparada. 


\section{RESULTADOS E DISCUSSAOO}

Com referência a anālise de crescimento do enxer to e do porta-enxerto da seringueira, realizada em $\mathrm{Pi}^{-}$ racicaba (SP), em quatro estágios de desenvolvimento, nas condições de viveiro, verifica-se pela Figura 1 que o enxerto de 'RRIM $600^{\prime}$ apresentou maiores variações em área foliar e em peso da matéria seca por ocasião do lançamento, no decorrer do 49 período, sendo que nos estagios anteriores mostrou menores VAP e VPS. O porta-enxerto de ' $T$ jir $16^{\prime}$ apresentou maiores variações em área foliar e peso da matéria seca no 39 período quan do se deu o lançamento, anteriormente ao enxerto. Do 19 ao 39 periodo observaram-se variações crescentes em $\mathrm{AF}$ e PS, as quais decresceram no 49 período. Os valores médios da VAF do enxerto ('RRIM 600') foram de $4,48 \mathrm{dm}^{2}$, sendo que do porta-enxerto atingiram $14,44 \mathrm{dm}^{2}$, nos períodos considerados. Considerando a média dos 4 períodos, a VPS foi de $7,92 \mathrm{~g}$ para o enxerto, sendo da ordem de $24,37 \mathrm{~g}$ para o cultivar Tjir 16 (porta-enxerto). Isto sugere o vigoroso desenvolvimento do porta-enxerto com relação ao cultivar enxertado. Também evidenciase uma possivel falta de compatibilidade entre o desenvolvimento de ambos.

A área foliar específica, capaz de refletir a espessura da folha, sendo a proporção relativa da super fície assimilatória e os tecidos mecânicos e condutores da folha, não revelou variações sensiveis entre o enxerto e o porta-enxerto, durante os períodos estudados (Figura 2). Observaram-se porém pequenas variações para o enxerto no decorrer dos quatro períodos. No caso do porta-enxerto, as variações foram maiores, na primeira amostragem obteve-se um baixo valor para a ārea foliar específica, quando a superfície assimilatória não apresentava ārea significativamente desenvolvida com relação ao peso do mesofilo, tecidos condutores e de sustentação da folha. Os valores médios da AFE de 'RRIM 600' mostraram-se da ordem de $1,7110 \mathrm{dm}^{2} \cdot \mathrm{g}^{-1}\left(\mathrm{AFE}_{1}\right)$ e de $1,6619 \mathrm{dm}^{2} \cdot \mathrm{g}^{-1}\left(\mathrm{AFE}_{2}\right)$, sendo que para ' $\mathrm{Tjir} 16^{\prime}$ foram de $1,7714 \mathrm{dm}^{2} \cdot \mathrm{g}^{-1}\left(\mathrm{AFE}_{1}\right)$, e de $1,9651 \mathrm{dm}^{2} \cdot \mathrm{g}^{-1}\left(\mathrm{AFE}_{2}\right)$. 

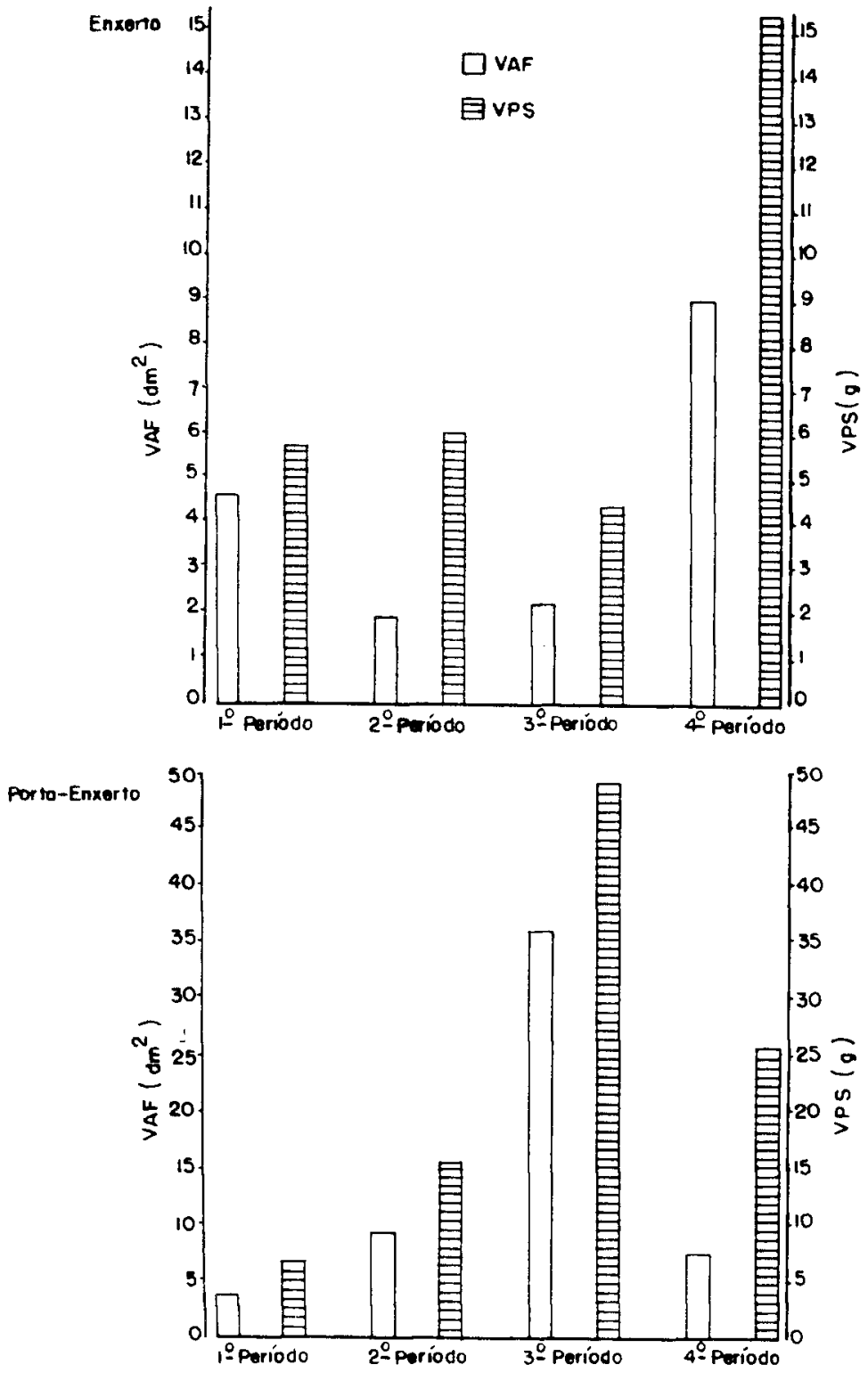

Fig. 1. Variações de ārea foliar (VAF) em $\mathrm{dm}^{2}$ e de peso da matéria seca (VPS) em g, do enxerto e do por ta-enxerto de seringueira, nos 4 períodos consi derados 


\section{DAFE, \\ 目 AFE $_{2}$}

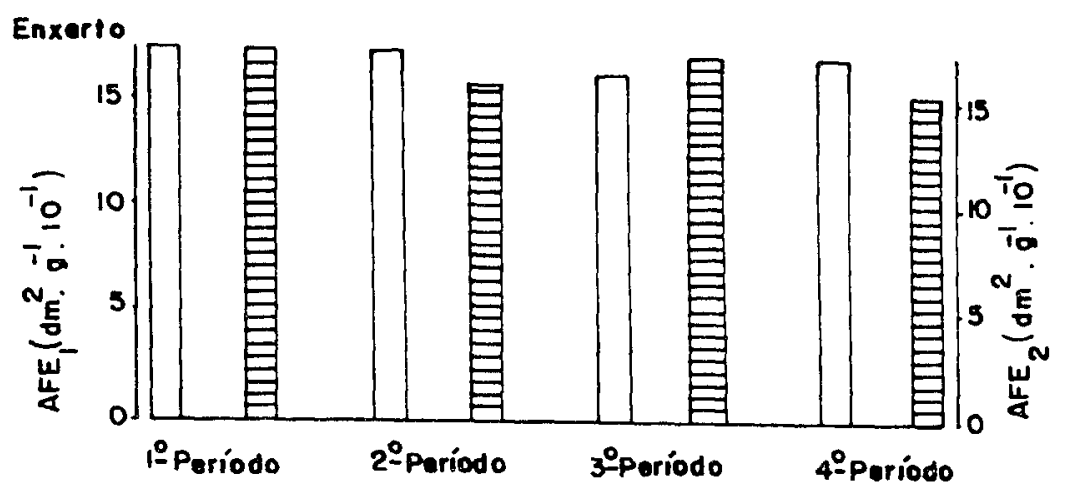

Porto-enxerto

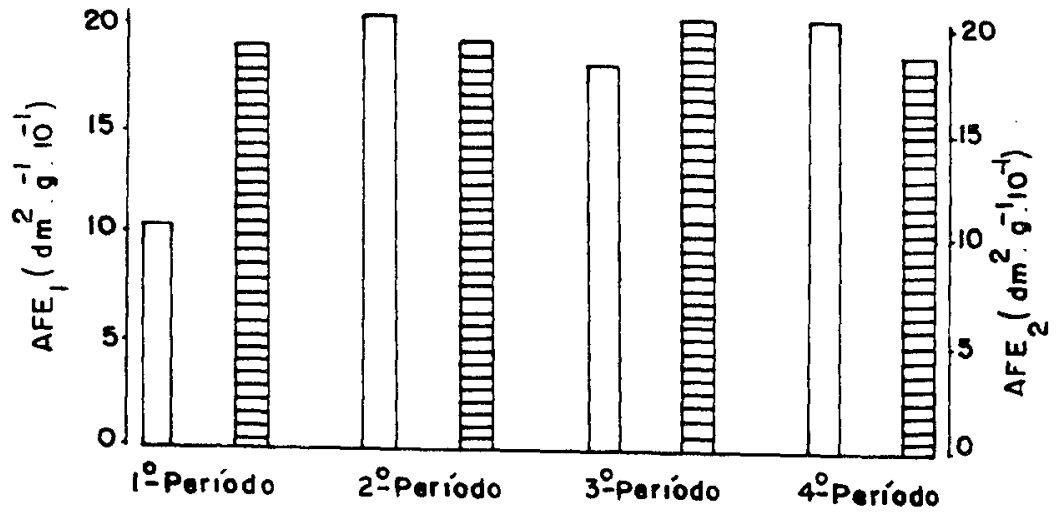

Fig. 2. Valores da ārea foliar específica (AFE) em $\mathrm{dm}^{2} \cdot \mathrm{g}^{-1}$, do enxerto e do porta-enxerto de seringueira, nos 4 periodos considerados 
A razão de ārea foliar, que relaciona a ārea foliar com o peso da matéria seca total da planta, apresentou valores ligeiramente mais altos no 19 período pa ra o enxerto, sendo que no porta-enxerto observaram-sé tendências de ligeira elevação nos 39 e 49 períodos (Fi gura 3). Isto sugere que o enxerto mostra maior proporção relativa de peso foliar no peso total da planta no início do desenvolvimento, sendo que o porta-enxerto revela esta tendência nos períodos finais. A RAF da seringueira 'RRIM 600' revelou valores médios de $0,8510 \mathrm{dm}^{2} \cdot \mathrm{g}^{-1}\left(\mathrm{RAF}_{1}\right)$ e de $0,7946 \mathrm{dm}^{2} \cdot \mathrm{g}^{-1}\left(\mathrm{RAF}_{2}\right)$, sendo que para 'Tjir $16^{\prime}$ foram de $0,4363 \mathrm{dm}^{2} \cdot \mathrm{g}^{-1}\left(\mathrm{RAF}_{1}\right)$ e de $0,5528 \mathrm{dm}^{2} \cdot \mathrm{g}^{-1}\left(\mathrm{RAF}_{2}\right)$, mostrando que a ärea foliar em re lação ao peso da matéria seca da planta é superior no enxerto comparativamente ao porta-enxerto. BLACKMAN \& WILSON (1951) encontraram os valores de $\operatorname{RAF}\left(\mathrm{dm}^{2} \cdot \mathrm{g}^{-1}\right)$ :

$\begin{array}{ll}\text { Iycopersicon esculentum } & 2,130 \\ \text { Solanum dulcamara } & 1,500 \\ \text { Pisum sativum } & 1,160 \\ \text { Vicia faba } & 0,750\end{array}$

Para algumas culturas, nas condições do estudo de São Paulo, encontrou-se os seguintes valores médios de $\operatorname{RAF}\left(\mathrm{dm}^{2} \cdot \mathrm{g}^{-1}\right)$ :

$\begin{array}{ll}\text { Arachis hypogaea } & 0,922 \\ \text { Lycopersicon esculentum } & 1,334 \\ \text { Gossypium hirsutum } & 0,442 \\ \text { Vigna unguiculata } & 0,960 \\ \text { Glycine max } & 1,394 \\ \text { Sorghum bicolor } & 1,495 \\ \text { Sacchamm spp. } & 0,641\end{array}$

Não somente a ārea foliar em termos absolutos é importante para a determinação do potencial de utilização mais eficiente da energia luminosa, mas também a proporção relativa entre tecidos fotossintetizantes e tecidos que apenas respiram. De acordo com WALTER (1971), quanto menor a proporção de partes não verdes 
$\square$ RAF,

目RAF 2
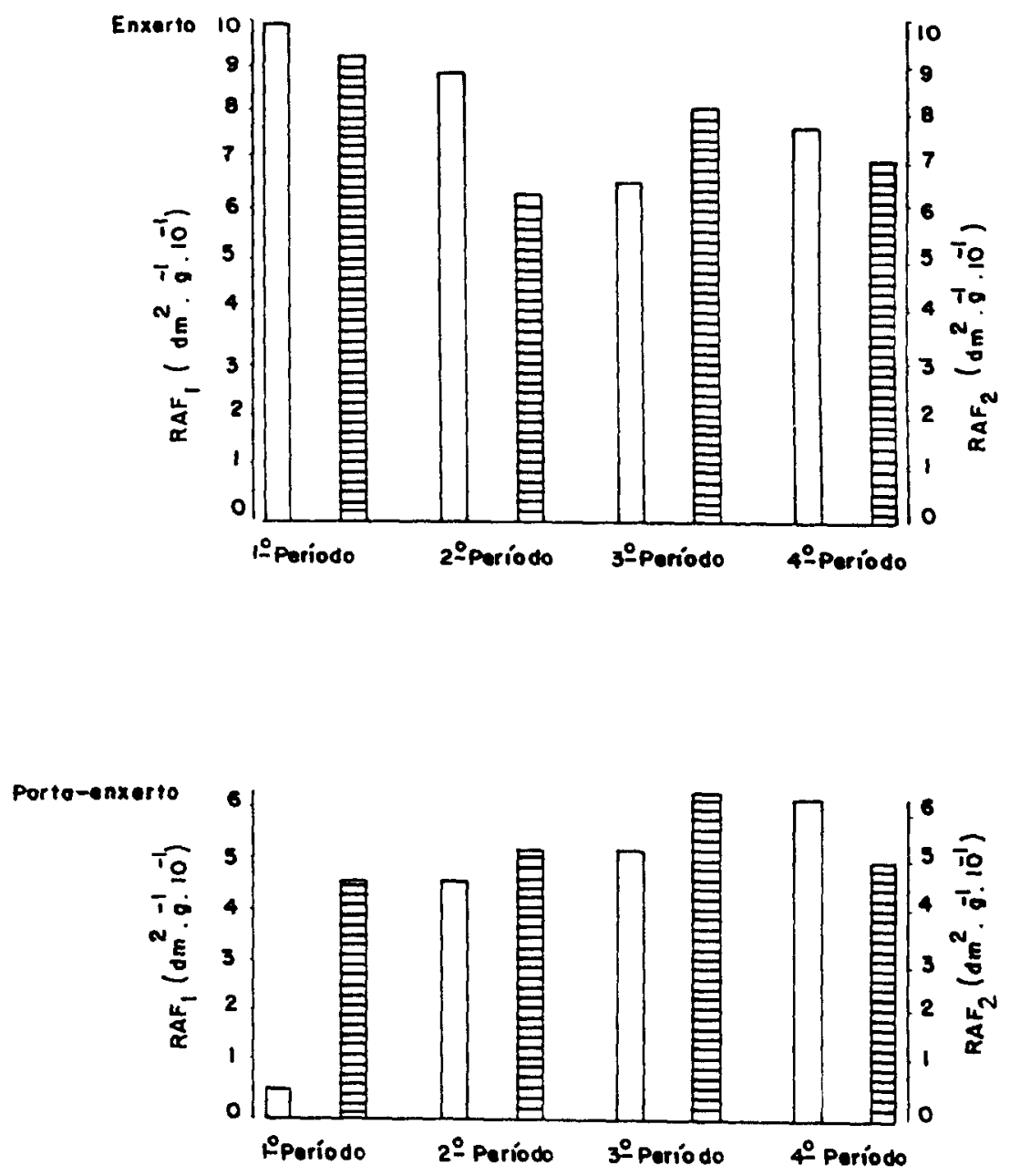

Fig. 3. Valores da razão de área foliar $(\mathrm{RAF}) \mathrm{em} \mathrm{dm}^{2} \cdot \mathrm{g}^{-1}$, do enxerto e do porta-enxerto de seringueira, nos 4 períodos considerados 
da planta, maior a sua tolerância ao sombreamento. Esta proporção é geralmente expressa em termos de RAF.

MONSELISE (1953) encontrou para limão 'Rugoso', lima 'Doce' e laranja 'Azeda' valores de RAF da ordem de $0,595,0,629$ e $0,770 \mathrm{dm}^{2} \cdot \mathrm{g}^{-1}$, respectivamente. GOODALL (1950) observou valores de 0,750 a $1,400 \mathrm{dm}^{2} \cdot \mathrm{g}^{-1}$, em cacaueiro. GRANGIER JR. \& ALVIM (1964) notaram RAF de 1,400 a $1,520 \mathrm{dm}^{2} \cdot g^{-1}$, na anālise de três cultivares de cacaueiro. De acordo com a Figura 3, o estudo da relação entre a ārea foliar e o peso da matéria seca total do enxerto mostrou valores mais altos no 19 periodo com relação aos demais. No caso do porta-enxerto, verificou-se uma tendência de valores crescentes do 19 ao 4 ? período, sendo que isto sugere uma participação crescente das folhas como orgãos receptores da energia solar, no contexto da estrutura total da planta.

A razão de peso foliar, que vem a ser a proporção relativa do peso foliar no peso total da planta, mostrou valores ligeiramente mais elevados no 19 período para o enxerto e tendências de ligeiro aumento nos 39 e 40 períodos no porta-enxerto (Figura 4). Esses resultados mostraram-se semelhantes àqueles obtidos para RAF. A RPF de 'RRIM 600' apresentou valores médios de $0,4840 \mathrm{~g} \cdot \mathrm{g}^{-1}\left(\mathrm{RPF}_{1}\right)$ e de $0,4878 \mathrm{~g} \cdot \mathrm{g}^{-1}\left(\mathrm{RPF}_{2}\right)$, sendo que para o cultivar Tjir 16 foram de $0,2635 \mathrm{~g} \cdot \mathrm{g}^{-1}\left(\mathrm{RPF}_{1}\right)$ e de $0,2798 \mathrm{~g} \cdot \mathrm{g}^{-1}\left(\mathrm{RPF}_{2}\right)$, indicando que a proporção do peso das folhas no peso total da planta é maior no enxerto do que no porta-enxerto.

Observou-se uma relação direta entre os valores da taxa de crescimento relativo e da taxa assimilatória líquida do enxerto e do porta-enxerto estudados (Figura 5). A taxa de crescimento relativo refere-se ao aumento em peso da matéria seca em relação ao peso inicial, por unidade de tempo, sendo que a taxa assimilatoria líquida trata da incorporação de assimilados fotossintēticos por unidade de ārea foliar e por unidade de tempo. Para o enxerto notaram-se valores decrescentes de TCR e TAL do 19 ao 39 período, enquanto no 

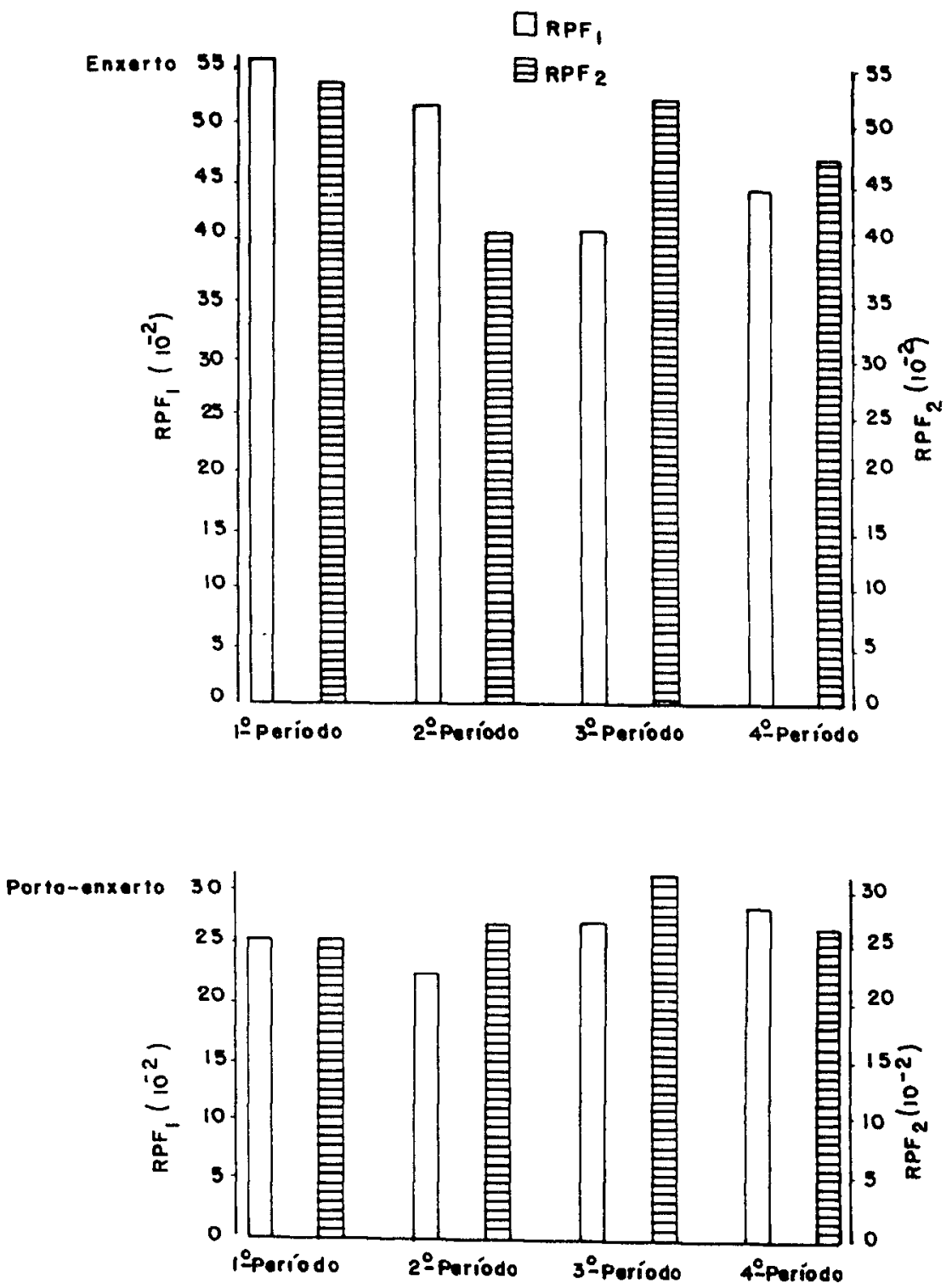

Fig. 4. Valores da razão de peso foliar (RPF) em $g \cdot g^{-1}$, do enxerto e do porta-enxerto de seringueira, nos 4 períodos considerados 

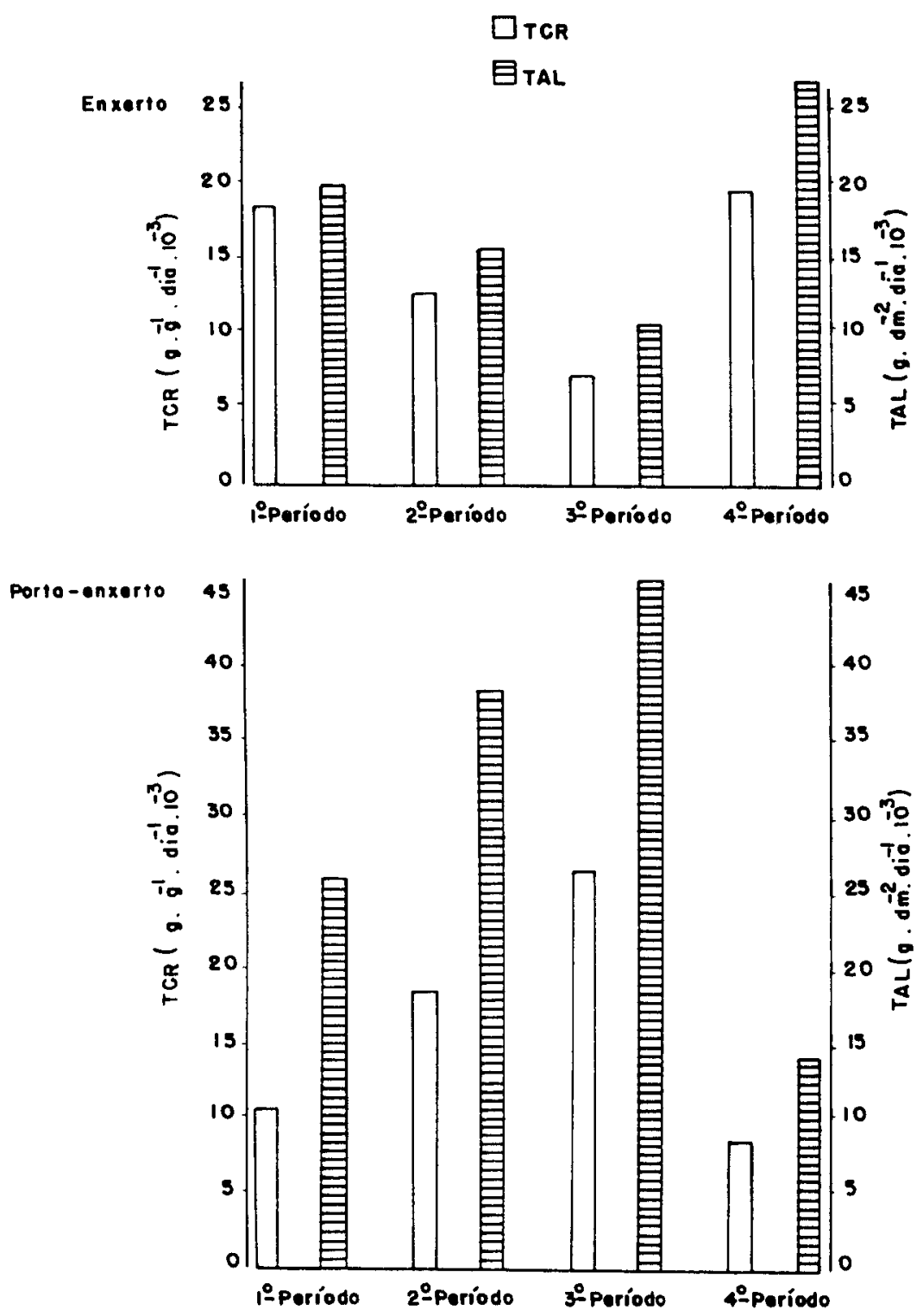

Fig. 5. Valores da taxa de crescimento relativo (TCR) em $g \cdot g^{-1}$ e da taxa assimilatōria líquida (TAL) em $\mathrm{g} \cdot \mathrm{dm}^{-2} \cdot \mathrm{dia}^{-1}$, do enxerto e do porta-enxerto de seringueira, nos 4 períodos considerados 
porta-enxerto observaram-se valores crescentes destes parâmetros no decorrer desses periodos. Para o enxerto verificou-se um incremento en TCR e TAL por ocasião do lançamento no 49 período. Como o lançamento do portaenxerto deu-se durante o 39 período, notou-se decréscimo nestes parâmetros no último período. Os valores médios da TCR do cultivar RRIM 600 mostraram-se da ordem de $0,0145 \mathrm{~g} \cdot \mathrm{g}^{-1} \cdot \mathrm{dia}^{-1}$, sendo que do cultivar $\mathrm{Tj}$ ir 16 foram de $0,0165 \mathrm{~g} \cdot \mathrm{g}^{-1} \cdot \mathrm{dia}^{-1}$. Esses resultados sugerem que os cultivares não mostraram diferenças elevadas nos incrementos de matéria seca por dia. Os dados obtidos para seringueira revelaram-se inferiores àqueles conseguidos para o cacaueiro, em Uruçuca $\left(0,023 \mathrm{~g} \cdot \mathrm{g}^{-1} \cdot \mathrm{dia}^{-1}\right)$, que por sua vez mostram-se menores do que as TCR observadas em soja $\left(0,066 \mathrm{~g} \cdot \mathrm{g}^{-1} \cdot \mathrm{dia}^{-1}\right)$ e em cana-de-açūcar $\left(0,118 \mathrm{~g} \cdot \mathrm{g}^{-1} \cdot \mathrm{dia}^{-1}\right)$, ambos em Piracicaba (Tabela 1). Con sidera-se que as espécies tolerantes ao sombreamentó apresentam um crescimento característico mais lento em relação às intolerantes, devido às suas taxas metabōlicas mais baixas (GRIME, 1965). A taxa assimilatória líquida que demonstra a incorporação de assimilados fotossintëticos por unidade de ārea foliar e por unidade de tempo, mostrou valores médios de $0,018 \mathrm{~g} \cdot \mathrm{dm}^{-2} \cdot \mathrm{dia}^{-1}$ para o enxerto, e de $0,031 \mathrm{~g} \cdot \mathrm{dm}^{-2} \cdot \mathrm{dia}^{-1}$ para o porta-enxerto. Esses resultados revelam que a TAL da seringueira mostra-se superior à do cacaueiro, da ordem de $0,015 \mathrm{~g} \cdot \mathrm{dm}^{-2} \cdot \mathrm{dia}^{-1}$ e $0,017 \mathrm{~g} \cdot \mathrm{dm}^{-2} \cdot \mathrm{dia}^{-1}$, em Uruçuca e Turrialba, respectivamente (Tabela 1 ).

\section{CONCLUSOEES}

Valores da variação de peso da matéria seca e da variação de área folíar demonstram o maior vigor do porta-enxerto com relação ao enxerto e uma certa incompatibilidade no período de desenvolvimento de ' $T$ jir 16' em relação ao 'RRIM 600 '.

A razão de ārea foliar e a razão de peso foliar revelam, respectivamente, uma maior proporção relativa da área e do peso seco foliar no peso total da planta 
Tabela 1. Taxa assimilatória líquida (TAL) e taxa de crescimento relativo (TCR) de algumas espécies cultivadas em diferentes regiões

\begin{tabular}{|c|c|c|c|c|}
\hline Cultivo & $\frac{\text { TAL }}{\left(\mathrm{g} \cdot \mathrm{dm}^{-2} \cdot \mathrm{dia}^{-1}\right)}$ & $\begin{array}{c}\mathrm{TCR} \\
\left(\mathrm{g} \cdot \mathrm{g}^{-2} \cdot \mathrm{dia}^{-1}\right)\end{array}$ & Local & Autor* \\
\hline Algodoeiro & 0,088 & 0,056 & Lima & Alvim \\
\hline Cacaueiro & 0,017 & 0,025 & Turrialba & Alvin \\
\hline $\begin{array}{l}\text { Cafeeiro } \\
\text { 'Tipica' }\end{array}$ & 0,024 & 0,029 & I ingo Maria & Alvim \\
\hline $\begin{array}{l}\text { Cafeeiro } \\
\text { 'Bourbon' }\end{array}$ & 0,025 & 0,034 & Tingo Maria & Alvim \\
\hline $\begin{array}{l}\text { Cafeeiro } \\
\text { 'Caturra' }\end{array}$ & 0,023 & 0,032 & Tingo Maria & Alvim \\
\hline Cana-de-Açücar & 0,097 & 0,034 & Paramonga & Alvim \\
\hline Cacaueiro & 0,015 & 0,023 & Uruçuca & Alvim \\
\hline Manıneira & 0,081 & 0,023 & Ibadan & Njoku \\
\hline Milho & 0,176 & 0,031 & Ibadan & Njoku \\
\hline Tomateiro & 0,115 & 0,033 & Ibadan & Njoku \\
\hline Amendoinzeiro & 0,077 & 0,046 & Jaboticabal & Castro \\
\hline Tomateiro & 0,102 & 0,132 & Piracicaba & Castro \\
\hline Algodoeiro & 0,063 & 0,062 & Piracicaba & Castro \\
\hline $\begin{array}{l}\text { Caupi } \\
\text { 'Epace } 8 \text { ' }\end{array}$ & 0,063 & 0,065 & Piracicaba & Castro \\
\hline Soja & 0,065 & 0,066 & Piracicaba & Castro \\
\hline $\begin{array}{l}\text { Sorgo Sacarino } \\
\text { 'BR } 505^{\prime}\end{array}$ & 0,125 & 0,005 & Piracicaba & Castro \\
\hline Cana-de-Açūcar & 0,167 & 0,118 & Piracicaba & Castro \\
\hline
\end{tabular}

* FONTE: Alvim e Njoku citados em ALVIM (1962) 
no início do desenvolvimento do enxerto, sendo que o porta-enxerto apresenta essa tendéncia mais tardiamente.

Hả uma relação direta entre os valores da taxa de crescimento relativo e da taxa assimilatória líquida do enxerto e do porta-enxerto, sendo que os cultivares não mostram diferenças sensiveis nos incrementos de matéria seca por unidade de tempo.

\section{REFERENCIAS BIBLIOGRAFICAS}

ALVIM, P.T. Los factores de la productividad agricola. In: CURSO INTERNACIONAL DE BASES FISIOLŌGICAS DE LA PRODUCCION AGRICOLA, Lima, 1962. Lima, IICA, 1962. $20 \mathrm{p}$.

BLACKMAN, G.E. \& WILSON, G.L. Physiological studies in the analysis of plant environment. VII. An analysis of the differential effects of light intensity on the net assimilation leaf area ratio and relative growth rate different species. Annals of Botany, Lond on, 15:373-408, 1951.

GOODALL, D.W. Growth analysis of cacao seedlings. Annals of Botany, London, 14:291-306, 1950.

GRANGIER JR., A. \& ALVIM, P.T. Análise de crescimento e do vigor híbrido em plântulas de cacau Catongo. In: CONGRESSO DA SOCIEDADE BOTÂNICA DO BRASIL, 15., Porto Alegre, 1964. Anais. Porto Alegre, 1964. 427-37.

GRIME, J.P. Shade tolerance in flowering plants. Nature, London, $5006(208): 161-3,1965$.

GUTIERREZ G.,J. \& JIMENEZ S., E. Desarrollo de um metodo fisiologico para la seleccion temprana de plantas perennes de alta productividad. Cacao, Turrialba, $12(1): 1-8.1967$.

MONSELISE, S.P. Growth analysis of citrus seedlings. II. A comparison between sweet lime, rough lemon and 
An.ESALQ, Piracicaba, 47 (parte 1):29-45, 1990

sour orange. Palestine Joumal of Botany, Rehovot, $8: 125-32$. 1953 .

MORAES, V.H.F. \& VALOIS, A.C.C. Produção de sementes clonais ilegitimas para porta-enxertos de seringueira (Hevea spp.). Comunicado Técnico. EMBRAPA/CNP Seringueira e Dendê, Manaus, (9):1-8, 1979.

SANTOS, P.M. Efeito da interação enxerto $x$ porta-enxer to em seringueira (Hevea spp.). Piracicaba, 1982. 66p. (Mestrado - Escola Superior de Agricultura"Luiz de Queiroz"/USP).

VALOIS, A.C.C.; PINHEIRO, E.; CONCEIÇÃO, H.E.D. \& SILVA, M.N.C. Competição de porta-enxertos de seringueira (Hevea spp.) e estimativas de parâmetros genéticos. Pesquisa Agropecuária Brasizeira, Brasília, 13(2): 49-54. 1978.

WALTER, H. Ecology of tropical and subtropical vegetation. Edinburg, O1iver \& Boyd, 1971. cap.1, p. $1-29$.

Entregue para publicação em 02/02/90 Aprovado para publicação em 26/04/90 